\title{
Abnormal Contractile Function due to Induction of Nitric Oxide Synthesis in Rat Cardiac Myocytes Follows Exposure to Activated Macrophage-conditioned Medium
}

\author{
Jean-Luc Balligand, * Dan Ungureanu, “ Ralph A. Kelly, “ Lester Kobzik, ” \\ David Pimental, * Thomas Michel, * and Thomas W. Smith* \\ Cardiovascular Division, Departments of *Medicine and ${ }^{\ddagger}$ Pathology, Brigham and Women’s Hospital and Harvard Medical School, \\ Boston, Massachusetts 02115
}

\begin{abstract}
The mechanism by which soluble mediators of immune cell origin depress myocardial contractility, either globally as in systemic sepsis, or regionally in areas of inflammatory myocardial infiltrates, remains unclear. When freshly isolated ventricular myocytes from adult rat hearts were preincubated for at least $24 \mathrm{~h}$ in medium conditioned by endotoxin (LPS)-activated rat alveolar macrophages, their subsequent inotropic response to the $\beta$-adrenergic agonist isoproterenol was reduced from $225 \pm 19 \%$ to $155 \pm 10 \%$ of the baseline amplitude of shortening (mean \pm SEM, $P<0.05$ ). Neither baseline contractile function nor the contractile response to high extracellular calcium were affected. To determine whether an endogenous nitric-oxide (NO)-signaling pathway within ventricular myocytes was responsible for their decreased responsiveness to isoproterenol, the L-arginine analogue L-NMMA was added to the preincubation medium. While L-NMMA did not affect baseline contractile function or the response of control myocytes to isoproterenol, it completely restored the positive inotropic response to isoproterenol in myocytes preincubated in LPS-activated macrophage medium. Release of $\mathrm{NO}$ by ventricular myocytes following exposure to activated macrophage medium was detected as an increase in cGMP content in a reporter-cell (RFL-6) bioassay and also as increased nitrite content in myocyte-conditioned medium. Thus, the depressed contractile response of adult rat ventricular myocytes to $\beta$-adrenergic agonists by a 24-h exposure to soluble inflammatory mediators is mediated at least in part by induction of an autocrine NO signaling pathway. (J. Clin. Invest. 1993. 91:2314-2319.) Key words: endotoxin • isoproterenol • septic shock • cytokine • microvascular endothelium
\end{abstract}

This paper was presented in abstract form at the 65th Scientific Sessions of the American Heart Association, November 1992, New Orleans, LA.

Address correspondence to Thomas W. Smith, M.D., Cardiology Division, Brigham and Women's Hospital, 75 Francis Street, Boston, MA 02115.

Received for publication 7 December 1992 and in revised form 29 January 1993.

J. Clin. Invest.

(c) The American Society for Clinical Investigation, Inc. 0021-9738/93/05/2314/06 \$2.00

Volume 91, May 1993, 2314-2319

\section{Introduction}

Cellular elements of the immune system have long been suspected to play a role in mediating the global myocardial dysfunction characteristic of septic shock, cardiac allograft rejection, and some forms of idiopathic cardiomyopathy (1). However, recent work indicates that direct cell-mediated cytotoxicity is not required to induce myocardial depression in experimental models of sepsis in animals or contractile dysfunction in isolated ventricular myocytes exposed to inflammatory mediators, including sera from patients with septic shock (2-5). Cell-free supernatants obtained from activated lymphocyte or macrophage cultures reversibly inhibit the expected increase in cAMP and concomitant positive inotropic responses of ventricular myocytes to the $\beta$-adrenergic agonist isoproterenol, but they have no effect on basal cAMP levels or on baseline contractile function $(2,3)$. This effect of activated immune-cell-conditioned medium on myocyte responsiveness to $\beta$ agonists is not immediate, but requires hours to become apparent (1).

Several cytokines that are known to be present in medium conditioned by activated immune cells have been shown to induce the synthesis of isoform(s) of nitric oxide (NO) in a number of cell types and tissues (6-9). NO is now recognized to be a nearly ubiquitous autocrine and paracrine chemical messenger, with several biologic activities including the activation of soluble intracellular guanylate cyclase $(10,11)$. Recent evidence from this laboratory has documented a role for an endogenous, constitutively present, NO-signaling pathway in regulating the physiologic responsiveness of neonatal and adult rat ventricular myocytes to muscarinic cholinergic and $\beta$ adrenergic agonists, respectively (12). Addition of analogues of L-arginine that act as specific inhibitors of NO synthase reproducibly enhanced the positive inotropic response to isoproterenol, but had no effect on baseline contractile function of freshly isolated adult rat ventricular myocytes paced at $1 \mathrm{~Hz}$. These data indicate that activation of a constitutive isoform of NO synthase within myocytes results in a countervailing autocrine effect that rapidly attenuates the effect of $\beta$-adrenergic stimulation in these cells. In addition, Schulz et al. have recently reported evidence, using biochemical assays of NO synthase activity, for both $\mathrm{Ca}^{2+}$-dependent constitutive, and $\mathrm{Ca}^{2+}$-independent, inducible isoforms of NO synthase in ventricular tissue slices and in isolated myocytes obtained from ventricles of adult rats, although no functional consequence of the increased synthesis of NO, such as contractility, was exam- 
ined (13). Increased activity of the $\mathrm{Ca}^{2+}$-independent isoform of NO synthase could be induced by a 24-h incubation with recombinant human TNF $\alpha$ and IL-1 $\beta$, two cytokines known to be present in activated immune-cell supernatants $(1,14)$.

In this report, freshly isolated adult rat ventricular myocytes, when incubated for $24 \mathrm{~h}$ in cell-free supernatant from endotoxin (LPS)-activated alveolar macrophages, were noted to exhibit a diminished inotropic response to the $\beta$-adrenergic agonist isoproterenol. This could be prevented by addition of the $\mathrm{L}$-arginine analogue $\mathrm{N}$-monomethyl-L-arginine ( $\mathrm{L}$ NMMA) ${ }^{1}$ to the myocyte incubation medium, implicating the induction of an endogenous $\mathrm{NO}$ signaling pathway in ventricular myocytes that blunts $\beta$-adrenergic inotropic responsiveness.

\section{Methods}

Isolation and preparation of adult rat ventricular myocytes. Calciumtolerant adult rat ventricular myocytes were isolated using the approach originally described by Claycomb and Palazzo (15), modified to limit the number of nonmyocyte cells present in primary isolates, as previously described $(16,17)$. Briefly, hearts were removed from etheranesthetized adult male Sprague-Dawley rats (225-250 g) and retrogradely perfused via the aorta for 5 min with a Krebs-Henseleit bicarbonate (KHB) buffer (16). Hearts were then perfused with nominally $\mathrm{Ca}^{2+}$-free $\mathrm{KHB}$ for 2-3 min, followed by an additional $20 \mathrm{~min}$ with nominally $\mathrm{Ca}^{2+}$-free $\mathrm{KHB}$ containing collagenase (Worthington Biochem. Corp., Freehold, NJ) and hyaluronidase (Sigma Immunochemicals, St. Louis, MO) ("enzyme buffer I"). Ventricular tissue was isolated, minced, and incubated in enzyme buffer I with added trypsin and deoxyribonuclease, filtered and washed five times in a 3:1 mixture of $\mathrm{Ca}^{2+}$-free $\mathrm{KHB}$ with a defined medium (i.e., ACCITT medium; see below) for short-term culture of myocytes (described below). Ventricular myocytes were separated from nonmyocyte cells by repetitive density gradient sedimentation steps, followed by differential attachment to laminin-coated tissue culture plates, as previously described (16). The composition of the defined medium used in these studies is a modification of that originally described by Volz et al. $(18,19)$, and consists of DME with Dulbecco's phosphate-buffered saline, including $25 \mathrm{mM}$ Hepes and $\mathrm{NaHCO}_{3}$ with L-glutamine (GIBCO BRL, Gaithersburg, MD), supplemented with $2 \mathrm{mg} / \mathrm{ml} \mathrm{BSA}, 2 \mathrm{mM}$ L-carnitine, 5 $\mathrm{mM}$ creatine, $0.1 \mu \mathrm{M}$ insulin, $5 \mathrm{mM}$ taurine and $10^{-10} \mathrm{M} \mathrm{T}_{3}$ (referred to as ACCITT medium) with $100 \mathrm{IU} / \mathrm{ml}$ penicillin and $100 \mu \mathrm{g} / \mathrm{ml}$ streptomycin (GIBCO BRL).

Preparation of rat alveolar macrophages. Alveolar macrophages were obtained by tracheal lavage using modifications of the technique described by Brain and Frank (20). Briefly, male Sprague-Dawley rats $(250-275 \mathrm{~g})$ were anesthetized with sodium pentobarbital $(50 \mathrm{mg} / \mathrm{kg})$. The trachea was cannulated, and the lungs lavaged repetitively with 5 -ml aliquots of sterile, ice-cold, phosphate-buffered saline containing $0.6 \mathrm{mM}$ ethylenediamine tetracetic acid (EDTA), $\mathrm{pH}$ 7.4. Bronchial lavage-derived suspensions of macrophages from six to eight rats were centrifuged at $700 \mathrm{rpm}$ (Centra-7R Tabletop Centrifuge; International Equipment Corp., Needham Heights, MA) for $10 \mathrm{~min}$, washed once with DME containing $25 \mathrm{mM}$ Hepes, L-glutamine, and pyruvate (GIBCO BRL), and pooled. Cells were resuspended at a concentration of $0.5 \times 10^{6} \mathrm{cells} / \mathrm{ml}$ in endotoxin-free DME containing $0.1 \%$ ( vol/ vol) albumin (bovine fraction V; Sigma Immunochemicals), $100 \mathrm{U} /$ $\mathrm{ml}$ penicillin and $100 \mathrm{U} / \mathrm{ml}$ streptomycin ("macrophage medium"), and cultured at a density of $2.5 \times 10^{6}$ cells $/ 60 \mathrm{~mm}$ dish at $37^{\circ} \mathrm{C}$ in $95 \%$ $\mathrm{O}_{2}, 5 \% \mathrm{CO}_{2}$. Over $95 \%$ of cells were viable as assessed by exclusion of trypan blue. $2 \mathrm{~h}$ after plating, culture dishes were washed to remove nonadherent cells, macrophages were exposed to either endotoxin, the LPS component of Salmonella typhimurium (87F402, Sigma Immuno-

1. Abbreviations used in this paper: KHB, Krebs-Henseleit bicarbonate; L-NMMA, L-arginine analogue $\mathrm{N}$-monomethyl-L-arginine. chemicals) at a concentration of $10 \mu \mathrm{g} / \mathrm{ml}$ (i.e., "LPS [+]") or saline (i.e., "LPS [-]") for $24 \mathrm{~h}$. Macrophage-conditioned medium was collected, centrifuged at $1,500 \mathrm{~g}$ for $10 \mathrm{~min}$ to remove cell debris, and then stored at $-70^{\circ} \mathrm{C}$ for further use.

Measurement of myocyte contractile function. Measurement of the amplitude and velocity of unloaded ventricular myocyte shortening and relengthening was made on the stage of an inverted phase-contrast microscope (Diavert; E. Leitz, Inc., Rockleigh, NJ) using an opticalvideo system in which the analogue motion signal was digitized and analyzed by computer, as previously described $(21,22)$. Coverslips with attached cells were placed in a temperature-controlled chamber at $37^{\circ} \mathrm{C}$ ( total volume, $5 \mathrm{ml}$ ) and continuously superfused at $0.8 \mathrm{ml} / \mathrm{min}$ with KHB buffer, with supplements as noted. One cell per coverslip was used.

Measurement of NO production. A rat lung fibroblast cell line (RFL-6 cells; American Type Culture Collection, Rockville, MD) served as reporter cells for NO production by virtue of its NO-sensitive guanylate cyclase activity, as previously described (12), a modification of the technique originally described by Ishii et al. (23). Freshly dissociated adult rat ventricular myocytes were plated at $2 \times 10^{5}$ cells $/$ well in 6-well plates (Costar Corp., Cambridge, MA) and incubated in ACCITT medium for a subsequent $24 \mathrm{~h}$ with or without $50 \%$ ( $\mathrm{vol} / \mathrm{vol}$ ) macrophage-conditioned media before assay of NO production. Myocytes were gently washed with $2 \mathrm{ml}$ of buffer containing ( $\mathrm{mM}$ ): 130 $\mathrm{NaCl}, 5 \mathrm{KCl}, 10$ glucose, $1 \mathrm{MgCl}_{2}, 1.5 \mathrm{CaCl}_{2}, 25$ Hepes ( $\mathrm{pH} 7.4$ ), plus added L-arginine ( $5 \mathrm{mM}$ ), and incubated in the absence of macrophage-conditioned medium for $20 \mathrm{~min}$, with addition of superoxide dismutase $(100 \mathrm{U} / \mathrm{ml}$ ) for the final $5 \mathrm{~min}$. An aliquot of myocyte-conditioned medium containing superoxide dismutase was then removed $(800 \mu \mathrm{l})$ and added directly to RFL-6 cells for a further 3-min incubation at $37^{\circ} \mathrm{C}$. The assay was terminated by aspiration of the RFL- 6 cell incubation medium and addition of $1 \mathrm{ml}$ of ice-cold $0.1 \mathrm{~N} \mathrm{HCl}$ to each well. After $20 \mathrm{~min}$ on ice, samples were neutralized with sodium acetate and sodium hydroxide and stored at $-20^{\circ} \mathrm{C}$. RFL- 6 cell-associated cGMP was determined by radioimmunoassay, as previously described (12).

Nitrite content of myocyte-conditioned medium was determined using established techniques (24). Ventricular myocytes $\left(2.8 \times 10^{5}\right.$ cells/well) were incubated for $24 \mathrm{~h}$ in $1.5 \mathrm{ml}$ ACCITT prepared from phenol red-free DME (GIBCO BRL) with or without macrophageconditioned medium ( $50 \%$, vol/ vol). After aspiration, the myocyteconditioned medium was centrifuged at $3,000 \mathrm{~g}$ for $15 \mathrm{~min}$, and nitrite content was measured in the supernatant. To $150 \mu$ l of cell supernatant, $900 \mu \mathrm{l}$ Griess reagent ( $0.75 \%$ sulfanilamide [ final concentration] in $0.5 \mathrm{~N} \mathrm{HCl} / 0.075 \%$ napthylethylenediamine) were added, and absorbance at $543 \mathrm{~nm}$ of the resulting chromophore was determined spectrophotometrically. A standard curve was constructed with known concentrations of sodium nitrite over the linear range of the assay $(0.1-$ $50 \mu \mathrm{M}$ nitrite), and the results are expressed as nmol of nitrite produced per $10^{6}$ cells. Nitrite release from myocytes was determined by subtracting the nitrite content of control medium (i.e., ACCITT) or macrophage-conditioned medium (i.e., ACCITT diluted 50\% (vol/ vol ) with LPS [+] medium) from the nitrite content of adult rat ventricular myocyte-conditioned medium.

Statistics. Data are expressed for convenience as mean \pm SEM, although no assumption was made concerning the distribution of the collected data. Statistical analysis of myocyte contractile function was performed using nonparametric tests on STATVIEW II ${ }^{\text {TM }}$ (Abacus Concepts Inc., Berkeley, CA) software. Comparison between two groups was performed using the Mann-Whitney test. Comparison among several nonrelated groups was performed using the KruskallWallis test followed by nonparametric multiple comparison tests for unequal sample sizes (25).

\section{Results}

Myocyte contractile function following exposure to macrophage-conditioned medium. Primary cultures of freshly iso- 
lated rat alveolar macrophages obtained by tracheal lavage were incubated for $24 \mathrm{~h}$ with endotoxin, in this case the LPS component of the cell wall of Salmonella typhimurium. After incubation with LPS, alveolar macrophage supernatants contained significant amounts of TNF $\alpha$, as assayed by WEHI clone 164 cytotoxicity (26) and IL-1, as assayed by stimulation of D10.64.1 T cell proliferation (27), indicative of endotoxininduced activation.

Exposure of primary isolates of adult-rat ventricular myocytes to alveolar macrophage-conditioned medium affected neither contractile amplitude (Fig. $1 A$ ) nor velocities of contraction or relaxation (data not shown) in the absence of isoproterenol compared with myocytes incubated in a defined medium (i.e., ACCITT) alone, whether or not the conditioned medium was obtained from macrophages preincubated with endotoxin. In preliminary experiments, a concentration of isoproterenol was chosen $(2 \mathrm{nM})$ that consistently produced a submaximal increase in contractile amplitude of between 150 and $200 \%$ of baseline values in myocytes stimulated at $2 \mathrm{~Hz}$ at $37^{\circ} \mathrm{C}$. Endotoxin alone had no effect on myocyte contractility, as preincubation of myocytes in ACCITT for $24 \mathrm{~h}$ with added LPS ( 10 $\mu \mathrm{g} / \mathrm{ml}$ ) had no effect on either baseline or on isoproterenol-stimulated amplitude of shortening in paced myocytes. The ratio of isoproterenol-stimulated contractile amplitude to basal contractile amplitude was $1.98 \pm 0.2$ vs. $1.92 \pm 0.19$ for control and endotoxin-pretreated myocytes, respectively ( mean $\pm \mathrm{SEM} ; P=\mathrm{NS} ; n=12$ cells per group from three myocyte preparations). Incubation of myocytes for $24 \mathrm{~h}$ in ACCITT diluted $50 \%$ ( $\mathrm{vol} / \mathrm{vol}$ ) with conditioned medium from alveolar macrophages not preincubated with LPS (i.e., LPS $[-])$ also had no effect on isoproterenol-stimulated con-

A

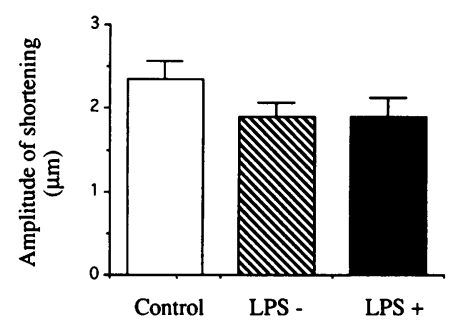

B

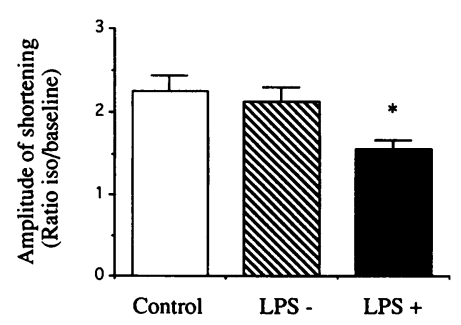

Figure 1. Effect of LPSactivated macrophageconditioned medium on contractile response to isoproterenol. Ventricular myocytes freshly isolated from adult rat hearts and attached to laminin-coated glass coverslips were preincubated in control-defined medium (open bar), or in control medium diluted $50 \%$ ( $\mathrm{vol} / \mathrm{vol})$ with medium conditioned by endotoxin-activated rat alveolar macrophages (i.e., LPS [+], solid bar), or by macrophages not previously exposed to endotoxin (i.e., LPS [-], hatched bar). ( $A$ ) After $24 \mathrm{~h}$, these media were removed, and baseline contractile function was determined in myocytes from each preincubation group, paced at $2 \mathrm{~Hz}$, and superfused with physiologic buffer at $37^{\circ} \mathrm{C}(n$ $=11-18$ myocytes in each group from five different experiments, mean \pm SEM, $P=$ NS). ( $B)$ The ratio of the amplitude of shortening following a submaximal $(2 \mathrm{nM})$ concentration of isoproterenol to the baseline amplitude of shortening is shown (mean \pm SEM, $n=11$ 18 myocytes from five different experiments, ${ }^{*} P<0.05$ for LPS [+] compared with LPS [-] or with control). tractile function when compared with contractile function of myocytes incubated in ACCITT alone. However, as shown in Fig. $1 B$, when myocytes were preincubated in medium containing supernatant from endotoxin-activated alveolar macrophage cultures (i.e., LPS $[+]$ ), there was a marked decline in the amplitude of contraction in response to isoproterenol, as well as comparable declines in the velocities of shortening and relengthening (data not shown). This diminished inotropic response to isoproterenol only became apparent after a minimum of 12 to $16 \mathrm{~h}$ of preincubation of myocytes with LPS-activated, macrophage-conditioned medium. Under the conditions employed here, dilutions of endotoxin-activated, macrophage-conditioned medium below $30 \%$ ( $\mathrm{vol} / \mathrm{vol}$ ) in ACCITT also did not affect myocyte contractile response to isoproterenol. The negative inotropic effect of LPS $(+)$ medium appeared to be specific for isoproterenol-treated cells, as the twofold increase in myocyte contractile amplitude induced by high extracellular calcium $(3.6 \mathrm{mM})$ was unaffected by prior exposure to medium conditioned by macrophages in the presence or absence of LPS ( ratio of isoproterenol-stimulated contractile amplitude to basal contractile amplitude, $2.05 \pm 0.67$ vs. $2.08 \pm 0.29$, respectively; mean \pm SEM; $n=12$ from three myocyte preparations; $P=\mathrm{NS}$ ).

$L-N M M A$ prevents negative inotropic effect of $L P S(+)$ medium. As the time of onset of the negative inotropic effect of LPS (+)-conditioned medium on myocyte responsiveness to isoproterenol was consistent with increased synthesis of an isoform of NO synthase in response to one or more inflammatory mediators produced by activated macrophages, the effect of the L-arginine analogue L-NMMA, an NO synthase inhibitor, was tested. Addition of L-NMMA ( $1 \mathrm{mM}$ ) for $24 \mathrm{~h}$ to ventricular myocytes maintained in either ACCITT alone, or in a $50 \%$ dilution in ACCITT of conditioned medium from alveolar macrophages not preincubated with LPS, had no effect on either baseline contractility (data not shown) or on the positive inotropic response to isoproterenol (Fig. 2). However, addition of L-NMMA to myocytes preincubated in a $50 \%$ dilution in ACCITT of LPS $(+)$ medium for $24 \mathrm{~h}$ restored the positive inotropic response of myocytes to isoproterenol (Fig. 2).

Induction of NO synthesis in ventricular myocytes by LPS(+) medium. To determine whether induction of NO synthesis in ventricular myocytes by inflammatory mediators could be measured directly in myocyte-conditioned medium, we used two techniques: a bioassay in which a reporter cell

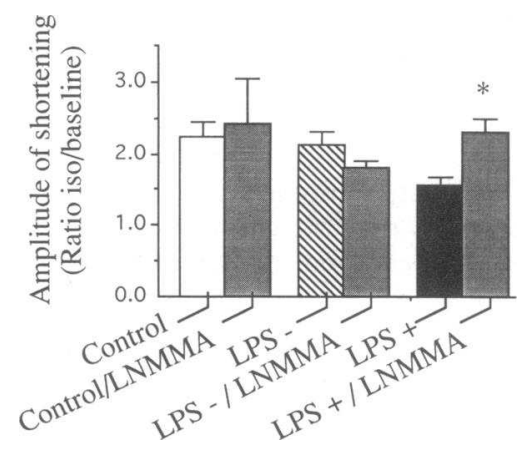

Figure 2. Effect of LNMMA on myocyte contractile response to isoproterenol. The ratio of the amplitude of shortening following 2 $\mathrm{nM}$ isoproterenol to the baseline amplitude of shortening is shown for myocytes preincubated for $24 \mathrm{~h}$ in control defined medium (open bar), control medium diluted $50 \%$ with

LPS $(+)$ (solid bar) or LPS $(-)$ (hatched bar)-conditioned media, either without or with $1 \mathrm{mM}$ L-NMMA (adjacent shaded bars) (mean \pm SEM, $n=8-16$ cells from three different experiments; ${ }^{*} P$ $<0.05$; LPS [+] with L-NMMA). 
responds to biologically active NO by increasing intracellular cGMP content, and a biochemical, colorimetric assay that measures total content of an oxidation product of NO, nitrite, in myocyte-conditioned medium. As shown in Fig. $3 \mathrm{~A}$, preincubation of myocytes with LPS $(+)$ medium increased their release of NO compared with myocytes preincubated with LPS $(-)$, as determined by a twofold increase in RFL- 6 cell cGMP content measured by radioimmunoassay. Similarly, the nitrite content of ventricular myocyte-conditioned medium following preincubation in control medium (i.e., ACCITT alone) was low. The nitrite content of medium conditioned by ventricular myocytes was markedly increased by preincubation with LPS $(+)$ medium (Fig. $3 B$ ). This increase was reversed by L-NMMA. As expected, based on recent reports documenting the role of $\mathrm{NO}$ as a cytotoxic agent released by activated macrophages in vivo and in vitro $(9,10), \operatorname{LPS}(+)$ medium itself contained significant amounts of nitrite. Activation of macrophages with LPS in an L-arginine-free macrophage medium reduced the nitrite content of this conditioned medium to barely detectable levels but had no effect on its ability to induce NO synthesis when added to ventricular myocytes in L-arginine replete medium (data not shown).

Of the cellular components of ventricular muscle, microvascular endothelial cells are the most abundant. As endothelium is now known to be an important target of immune cytokines, as well as itself an important source of cytokines and other potentially cardioactive peptides and autacoids, we also examined whether primary cultures of endothelial cells isolated from adult rat ventricular tissue and characterized as having a microvascular phenotype, as previously described (19), showed evidence of inducible NO production in response to incubation in LPS (+) medium. Cardiac microvascular endothelial cells, at

A

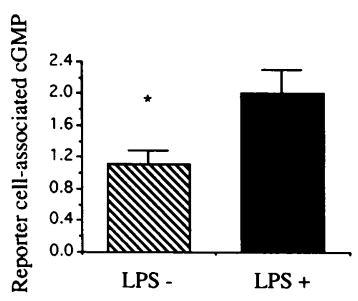

B

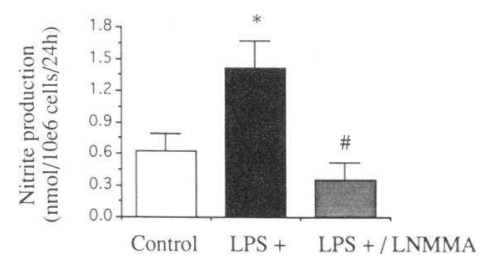

Figure 3. Increased release of NO by ventricular myocytes following preincubation with LPS $(+)$ medium. Both biologically active NO and an oxidation product of NO were measured in myocyte-conditioned medium by bioassay of intracellular cGMP content in RFL6 cells $(A)$ or by measurement of nitrite content in the medium $(B) .(A)$ The results in the RFL- 6 cells bioassay are normalized to the basal endogenous cGMP content of RFL6 cells not exposed to

myocyte-conditioned medium, which was $0.55 \pm 0.21 \mathrm{pmol} /$ well. Preincubation of myocytes with LPS $(+)$ medium induced a twofold increase in cGMP compared with control $\left({ }^{*}, P<0.05, n=9\right.$ from three separate experiments). (B) Nitrite content in myocyte-conditioned medium is expressed as $\mathrm{nmol} / 10^{6}$ cells $/ 24 \mathrm{~h}$, following subtraction of the nitrite content of control or macrophage-conditioned preincubation medium. Preincubation of myocytes with LPS $(+)$ medium markedly increased subsequent nitrite production, and this could be abrogated by addition of L-NMMA ( ${ }^{*}, P<0.05$; \#, $P<0.01$; mean $\pm \mathrm{SEM} ; n=11-14$ from three separate experiments). least under the culture conditions employed here, had low constitutive levels of $\mathrm{NO}$ release $(0.97 \pm 0.22 \mathrm{pmol} /$ well $)$, as measured by RFL- 6 cell cGMP content. NO production was markedly induced by a 24 -h preincubation in LPS $(+)$ medium, however, to $6.94 \pm 1.23 \mathrm{pmol} /$ well $($ mean $\pm \mathrm{SEM} ; n=7-14$ wells from three separate microvascular endothelial cell cultures; $P$ $<0.01)$.

\section{Discussion}

The effect of activated macrophage-conditioned medium on myocyte responsiveness to isoproterenol was not apparent for at least $12 \mathrm{~h}$, consistent with the induction of cytokine-responsive calcium-insensitive NO synthase activity in cardiac myocytes $(11,13)$. This is in contrast to recent data reported by Finkel et al. (28), who demonstrated that several recombinant cytokines, including TNF $\alpha$, IL-2, and IL-6, had negative inotropic effects within 2-3 min when added to superfusion medium bathing an isolated hamster-papillary-muscle preparation. This negative inotropic effect was apparent in the absence of any cardiotonic agents including adrenergic agonists and could be prevented by addition of L-NMMA to the superfusion medium. As the rapid onset of the negative inotropic effect is inconsistent with increased transcription and synthesis of a calcium-insensitive, inducible isoform of NO synthase, it is possible, as these authors suggest (28), that these cytokines resulted in activation of a calcium/calmodulin-responsive constitutive isoform of NO synthase in papillary muscle. While Finkel et al. (28) did not report any biochemical or bioassay data in support of NO synthesis by a constitutive isoform of the enzyme, Schultz et al. (13) did detect $\mathrm{Ca}^{2+}$-dependent synthesis of $\mathrm{L}-$ citrulline from L-arginine in freshly isolated myocytes from adult rat hearts, and we have reported constitutive production of bioactive NO from these cells (12).

Although we have also reported that addition of L-nitro-arginine (L-NA) increases the contractile response to isoproterenol by $30-35 \%$ within minutes in freshly isolated adult rat ventricular myocytes, presumably due to inhibition of a constitutive isoform of NO synthase, we have never observed an effect of NO synthase inhibitors in the absence of a $\beta$ agonist (12). L-NMMA, rather than $N$-nitro-L-arginine, was employed in the present study because of the marked differences in the sensitivity of constitutive and calcium-independent, inducible isoforms of NO synthase to inhibition by NG-monosubstituted arginine analogues $(29,30)$. This may explain why an increased inotropic response to isoproterenol with L-NMMA was not observed in this study in control myocytes preincubated in defined medium (i.e., ACCITT) alone. As reported by Chung et al. (31), a pertussis toxin-sensitive, GTP-binding protein appears to mediate the diminished inotropic response to isoproterenol of neonatal rat cardiocytes exposed to soluble inflammatory mediators. Whether these inflammatory mediators act via $\mathrm{a}_{\mathrm{i}}-$ or $\mathrm{G}_{\mathrm{o}}$-linked signal transduction pathway to induce NO synthesis in adult myocytes, or whether cytokineinduced NO synthesis within myocytes leads to $G_{i}-$ or $G_{0}-$ linked uncoupling of $\beta$-adrenergic receptors, is the subject of ongoing research and remains to be determined. Other mechanisms by which increased NO synthesis could affect myocyte contractile function, aside from discrete, specific effects on signal transduction pathways, have been described in other cell types (32-34).

Although many recombinant or synthetic cytokines and other autacoids known to be elaborated by activated macro- 
phages are now available, the use of medium conditioned by LPS-activated macrophages may provide more biologically relevant combinations and concentrations of inflammatory mediators, while obviating the potential problem of differential responsiveness of rat tissue to some recombinant cytokines based on the known murine or human peptide sequences. Using a different approach, Brady et al. (35) have recently demonstrated that $\mathrm{L}$-arginine analogues can reverse in part the decreased contractility exhibited by isolated adult guinea pig ventricular myocytes obtained from LPS-pretreated animals, while these analogues had no effect on baseline contractile function of myocytes obtained from control animals. While no biochemical or other bioassay data to confirm increased NO synthesis in ventricular myocytes from LPS-treated animals was given, these data are consistent with those reported in this manuscript linking induction of NO synthase by inflammatory mediators to depressed contractile function.

We believe that induction of NO synthase within the myocytes themselves was the source of NO responsible for the negative inotropic response to isoproterenol. The number of nonmyocyte cells present in primary cultures of adult rat ventricular myocytes in defined medium following our standard isolation procedure, which includes repetitive density gradient sedimentation steps as well as differential adhesion to laminincoated coverslips, is typically less than $5 \%$ at $24 \mathrm{~h}$. Also, since isolated myocyte contractile function was assayed by placing individual coverslips in a chamber in which myocytes were continually superfused with fresh KHB buffer, it is unlikely that NO released into the buffer from a small number of contaminating nonmyocyte cells could have been responsible for the blunting of the inotropic response to isoproterenol in myocytes preincubated with endotoxin-activated macrophage-conditioned medium. Nevertheless, the fact that microvascular endothelial cells isolated from adult rat ventricular tissue also respond to soluble inflammatory mediators in the endotoxinactivated alveolar macrophage-conditioned medium suggests that NO released by these cells may contribute to the contractile dysfunction of adjacent myocytes. The predicted mean diffusion distance of NO (under physiologic conditions $\sim 200$ $600 \mu \mathrm{m}[11]$, depending on, among other factors, adduct formation with cellular and interstitial proteins), suggests that NO released by endothelium in the microvasculature could affect subjacent tissue, particularly in highly vascular cardiac muscle where the ratio of microvessels to myofibrils is approximately 1:1 (36).

In summary, ventricular myocytes isolated from adult rat hearts exhibit a decreased contractile responsiveness to $\beta$ adrenergic agonists following exposure to cell-free supernatant from endotoxin-activated rat alveolar macrophages. This effect was reversed by specific inhibitors of NO synthase. Taken together with data reported previously (12), these findings suggest that ventricular myocytes contain both constitutive and cytokine-inducible NO synthase activities. Increased NO produced by myocytes, and perhaps by nonmyocyte cells including microvascular endothelial cells as well, may contribute to the contractile dysfunction characteristic of advanced systemic sepsis and some cardiomyopathies.

\section{Acknowledgments}

We thank Dr. Abul Abbas (Harvard Medical School) for his advice and for performing the D10.64.1 T cell proliferation assay for IL-1, and Dr. Chandana Saha for her excellent technical assistance.
This work was supported in part by grant HL36141 (to T. W. Smith) from the National Institutes of Health, a Clinician-Scientist Award (to T. Michel) from the American Heart Association, scholarships (to D. Ungureanu) from the Franco-American Commission, the Lavoisier Program, and the Simone and Gino del Duca Foundation, and a fellowship (to J.-L. Balligand) from the Belgian Fonds National de la Recherche Scientifique.

\section{References}

1. Lange, L. G., and G. F. Schreiner. 1992. Immune cytokines and cardiac disease. Trends Cardiovasc. Med. 2:145-151.

2. Gulick, T., M. K. Chung, S. J. Pieper, G. F. Schreiner, and L. G. Lange. 1988. Immune cytokine inhibition of $\beta$-adrenergic agonist stimulated cyclic AMP generation in cardiac myocytes. Biochem. Biophys. Res. Commun. 150:1-9.

3. Gulick, T., S. J. Pieper, M. A. Murphy, L. G. Lange, and G. F. Schreiner. 1991. A new method for assessment of cultured cardiac myocyte contractility detects immune factor-mediated inhibition of $\beta$-adrenergic responses. Circulation. 84:313-321.

4. Reilly, J. M., R. E. Cunnion, C. Burch-Whitman, M. M. Parker, J. H. Shelhamer, and J. E. Parrillo. 1989. A circulating myocardial depressant substance is associated with cardiac dysfunction and peripheral hypoperfusion (lactic acidemia) in patients with septic shock. Chest. 95:1072-1080.

5. Natanson, C., P. W. Eichenholz, R. L. Danner, P. Q. Eichacker, W. D. Hoffman, G. C. Kuo, S. M. Banks, T. J. MacVittie, and J. E. Parrillo. 1989. Endotoxin and tumor necrosis factor challenges in dogs simulate the cardiovascular profile of human septic shock. J. Exp. Med. 169:823-832.

6. Leone, A. M., R. J. J. Palmer, R. G. Knowles, P. L. Francis, D. S. Ashton, and S. Moncada. 1991. Constitutive and inducible nitric oxide syntheses incorporate molecular oxygen into both nitric oxide and citrulline. J. Biol. Chem. 266:23,790-23,795.

7. Lamas, S., T. Michel, B. M. Brenner, and P. A. Marsden. 1991. Nitric oxide synthesis in endothelial cells: evidence for a pathway inducible by TNF- $\alpha . A m$. J. Physiol. 261:C634-C641.

8. Scott-Burden, T., V. B. Schini, E. Elizondo, D. C. Junquero, and P. M Vanhoutte. 1992. Platelet-derived growth factor suppresses and fibroblast growth factor enhances cytokine-induced production of nitric oxide by cultured smooth muscle cells. Effects on cell proliferation. Circ. Res. 71:1088-1100.

9. Xie, Q.-W., H. J. Cho, J. Calaycay, R. A. Mumford, K. M. Swiderek, T. D. Lee, A. Ding, T. Troso, and C. Nathan. 1992. Cloning and characterization of inducible nitric oxide synthase from mouse macrophages. Science (Wash. DC). 256:225-228.

10. Lowenstein, C. J., and S. H. Snyder. 1992. Nitric oxide, a novel biologic messenger. Cell. 70:705-707.

11. Knowles, R. G., and S. Moncada. 1992. Nitric oxide as a signal in blood vessels. Trends Biochem. Sci. 17:399-402.

12. Balligand, J.-L., R. A. Kelly, P. A. Marsden, T. W. Smith, and T. Michel. 1993. Control of cardiac muscle cell function by an endogenous nitric oxide signalling system. Proc. Natl. Acad. Sci. USA. 90:347-351.

13. Schulz, R., E. Nava, and S. Moncada. 1992. Induction and potential biological relevance of a $\mathrm{Ca}^{2+}$-independent nitric oxide synthase in the myocardium. Br. J. Pharmacol. 105:575-580.

14. Arai, K., F. Lee, A. Miyajima, S. Miyatake, N. Arai, and T. Yokota. 1990. Cytokines: coordinators of immune and inflammatory responses. Annu. Rev Biochem. 59:783-836.

15. Claycomb, W. C., and M. C. Palazzo. 1980. Culture of the terminally differentiated adult cardiac muscle cell: $\mathrm{A}$ light and scanning electron microscope study. Dev. Biol. 80:466-482.

16. Eid, H., D. M. Larson, J. P. Springhorn, M. A. Attawia, R. C. Nayak, T. W. Smith, and R. A. Kelly. 1992. Role of epicardial mesothelial cells in the modification of phenotype and function of adult rat ventricular myocytes in primary coculture. Circ. Res. 71:40-50.

17. Springhorn, J. P., Ø. Ellingsen, H.-J. Berger, R. A. Kelly, and T. W. Smith. 1992. Transcriptional regulation in cardiac muscle. Coordinate expression of Id with a neonatal phenotype during development and following a hypertrophic stimulus in adult rat ventricular myocytes in vitro. J. Biol. Chem. 267:1436014365 .

18. Volz, A., H. M. Piper, B. Siegmund, and P. Schwartz. 1991. Longevity of adult ventricular rat heart muscle cells in serum-free primary culture. $\mathrm{J}$. Mol. Cell. Cardiol. 23:161-173.

19. Nishida, M., W. W. Carley, M. E. Gerritsen, Ø. Ellingsen, R. A. Kelly, and T. W. Smith. 1993. Isolation and characterization of human and rat cardiac microvascular endothelial cells. Am. J. Physiol. 264:H639-H652.

20. Brain, J. D., and R. Frank. 1968. Recovery of free cells from rat lungs by repeated washings. J. Appl. Physiol. 25:63-69.

21. Kelly, R. A., H. Eid, B. K. Kramer, M. O’Neill, B. T. Liang, M. Reers, and T. W. Smith. 1990. Endothelin enhances the contractile responsiveness of adult rat ventricular myocytes to calcium by a pertussis toxin-sensitive pathway. $J$. Clin. Invest. 86:1164-1171. 
22. Borzak, S., S. Murphy, and J. D. Marsh. 1991. Mechanisms of rate staircase in rat ventricular cells. Am. J. Physiol. 260:H884-H892.

23. Ishii, K., H. Sheng, T. D. Warner, U. Förstermann, and F. Murad. 1991. A simple and sensitive bioassay method for detection of EDRF with RFL-6 rat lung fibroblasts. Am. J. Physiol. 261:H598-H603.

24. Marsden, P. A., and B. J. Ballermann. 1990. Tumor necrosis factor $\alpha$ activates soluble guanylate cyclase in bovine glomerular mesangial cells via an L-arginine-dependent mechanism. J. Exp. Med. 172:1843-1852.

25. Hollander, M., D. A. Wolfe. 1973. Nonparametric Statistical Methods. John Wiley and Sons, Inc., New York. 138-162.

26. Espevik, T., and J. Nissen-Meyer. 1986. A highly sensitive cell line, WEH 164 clone 13, for measuring cytotoxic factor/tumor necrosis factor from human monocytes. J. Immunol. Methods. 95:99-105.

27. Kaye, J., S. Gillis, S. B. Mizel, E. M. Shevach, T. R. Malek, C. A. Dinarello, L. B. Lachman, and C. A. Janeway, Jr. 1984. Growth of a cloned helper T cell line induced by a monoclonal antibody specific for the antigen receptor: interleukin 1 is required for the expression of receptors for interleukin 2. J. Immunol. 133:1339-1345.

28. Finkel, M. S., C. V. Oddis, T. D. Jacob, S. C. Watkins, B. G. Hattler, and R. L. Simmons. 1992. Negative inotropic effects of cytokines on the heart mediated by nitric oxide. Science (Wash. DC). 257:387-389.

29. Gross, S. S., D. J. Stuehr, K. Aisaka, E. A. Jaffe, R. Levi, and O. W. Griffith. 1990. Macrophage and endothelial cell nitric oxide synthesis: cell-type selective inhibition by NG-aminoarginine, NG-nitroarginine and NG-methylarginine. Biochem. Biophys. Res. Commun. 170:96-103.
30. Gross, S. S., E. A. Jaffe, R. Levi, and R. G. Kilbourn. 1991. Cytokine-activated endothelial cells express an isotype of nitric oxide synthase which is tetrahydrobiopterin-dependent, calmodulin-independent and inhibited by arginine analogs with a rank-order of potency characteristic of activated macrophages. Biochem. Biophys. Res. Commun. 178:823-829.

31. Chung, M. K., T. S. Gulick, R. E. Rotondo, G. F. Schreiner, and L. G. Lange. 1990. Mechanisms of cytokine inhibition of $\beta$-adrenergic agonist stimulation of cyclic AMP in rat cardiac myocytes. Impairment of signal transduction Circ. Res. 67:753-763.

32. Dimmeler, S., F. Lottspeich, and B. Brüne. 1992. Nitric oxide causes ADP-ribosylation and inhibition of glyceraldehyde-3-phosphate dehydrogenase. J. Biol. Chem. 267:16,771-16,774.

33. Zhang, J., and S. H. Snyder. 1992. Nitric oxide stimulates auto-ADP-ribosylation of glyceraldehyde-3-phosphate dehydrogenase. Proc. Natl. Acad. Sci. USA. 89:9382-9385.

34. Geng, Y.-J., G. K. Hansson, and E. Holme. 1992. Interferon- $\gamma$ and tumor necrosis factor synergize to induce nitric oxide production and inhibit mitochondrial respiration in vascular smooth muscle cells. Circ. Res. 71:1268-1276.

35. Brady, A. J. B., P. A. Poole-Wilson, S. E. Harding, and J. B. Warren. 1992. Nitric oxide production within cardiac myocytes reduces their contractility in endotoxemia. Am. J. Physiol. 263:H1963-H1966.

36. Rakusan, K., M. F. Flanagan, T. Geva, J. Southern, and R. Van Praagh 1992. Morphometry of human coronary capillaries during normal growth and the effect of age in left ventricular pressure-overload hypertrophy. Circulation. $86: 38-46$. 